\section{Malnutrición por exceso: alta frecuencia de sobrepeso y obesidad en escolares chilenos con síndrome de Down}

\author{
LILY JIMÉNEZ ${ }^{1}$, JAIME CERDA², \\ GIGLIOLA ALBERTI ${ }^{3}$, MACARENA LIZAMA ${ }^{1,4}$
}

\section{High rates of overweight and obesity in Chilean children with Down syndrome}

\begin{abstract}
Background: Overweight and obesity in Down syndrome (DS) is a common problem. Chile has a high prevalence of DS. Aim: To determine overweight and obesity rates in students with DS and evaluate the concordance of three different growth charts. Material and Methods: Seventy nine students with DS aged between 6 and 18 years (56\% males), from three different schools, were included. Weight and height were measured and their body mass index (BMI) was calculated. The nutritional diagnosis was made according to BMI. Myrelid SDM/2002, National Center for Health Statistics (NCHS)/2000, World Health Organization (WHO)/2007 charts for people with DS were used. Results: Thirty percent of participants had hypothyroidism, $22.8 \%$ congenital heart disease and $5 \%$ asthma. Overweight and obesity rates according to SDM/2002, NCHS/2000 and $\mathrm{WHO} / 2007$ were 43, 57 and 66\% respectively. The concordance between WHO/2007 and NCHS/2000 was almost perfect, but not with SDM/2002. Conclusions: A high rate of overweight and obesity was found in this group of children with DS, independent of the charts used or their comorbidities.
\end{abstract}

(Rev Med Chile 2015; 143: 451-458)

Key words: Children; Down syndrome; Growth charts; Overweight; Obesity.

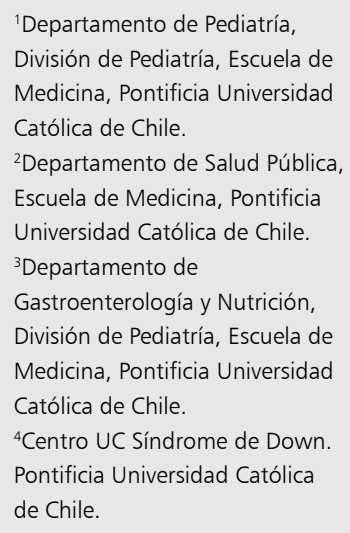

Recibido el 25 de junio de 2014, aceptado el 26 de enero de 2015

No hubo financiamiento para este trabajo.

Correspondencia a: Dra. Macarena Lizama Calvo Lira $85,5^{\circ}$ piso, Santiago-Chile. mlizama@med.puc.cl
$\mathrm{E}$ n Chile la tasa de nacimientos de niños con síndrome de Down (SD) es de 2,47 por mil nacidos vivos (NV) durante el período del 1998 al 2005', con ascenso progresivo en las últimas décadas, siendo la tasa más alta descrita en Latinoamérica, donde el promedio de nacimientos en la región es de 1,89 por mil NV ${ }^{1}$, según reporta el Estudio Colaborativo Latinoamericano de Malformaciones Congénitas (ECLAMC) ${ }^{1}$. En tanto, la malnutrición por exceso (MNE), que corresponde a niños con sobrepeso u obesidad, es un fenómeno creciente a nivel mundial, con una prevalencia el año 2012 en población latina de $40 \%$ en niños de 8 a 17 años en España ${ }^{2}$, en México ${ }^{3}$ de $34,4 \%$ en niños de 6 a 12 años y en Chile, el año 2011 alcanzó $22,1 \%$ de obesidad en escolares entre 6 y 7 años, según datos de la Junta Nacional de Auxilio
Escolar y Becas (JUNAEB) ${ }^{4}$, institución que recolecta los datos antropométricos de los escolares de primer año básico de escuelas municipalizadas y particulares subvencionados en Chile.

La prevalencia internacional de MNE en niños y jóvenes con SD se estima entre 32\% y 50\%, según las distintas series ${ }^{5-6}$ y en Chile, el estudio realizado por Pinheiro el año 2002, reporta 19,6\% MNE en niños con SD de 3 meses a 18 años $^{7} y$ de acuerdo a la cohorte descrita por Retamales en lactantes menores de 1 año, habría una tendencia a aumentar la frecuencia de MNE en la medida que aumenta la edad ${ }^{8}$.

Actualmente se desconoce la prevalencia de sobrepeso y obesidad en niños con SD en Chile, principalmente por la diversidad de patrones antropométricos utilizados para realizar el diag- 
nóstico nutricional en este grupo, ya sea con tablas para población general y/o curvas de crecimiento elaboradas en población con SD. La recomendación del programa nacional de salud de Down España, sugiere usar las curvas antropométricas publicadas por la Fundación Catalana de SD Peso/Edad y Talla/Edad ${ }^{9}$; por otra parte, en el año 2011, la Academia Americana de Pediatría (AAP), sugirió evaluar a los niños con SD con curvas de índice de masa corporal (IMC) según edad cronológica, usando el patrón del Centro Nacional de Salud y Estadísticas de Estados Unidos (NCHS por inglés: National Center for Health and Statistics) para población general, hasta tener curvas más actualizadas y específicas para población con $\mathrm{SD}^{10}$.

Siendo la MNE un problema emergente y en un país con la tasa más alta de nacimientos de niños con SD en Latinoamérica ${ }^{1}$, el propósito de este estudio es caracterizar la situación de salud nutricional en escolares con SD, evaluar la frecuencia de MNE según diferentes patrones antropométricos y el nivel de concordancia entre ellos, para generar recomendaciones de promoción y prevención en salud adecuados para este grupo vulnerable de nuestra sociedad.

\section{Pacientes y Métodos}

\section{Tipo de estudio}

Estudio de corte transversal, descriptivo, que evalúa la frecuencia de MNE (sobrepeso y obesidad) en escolares con SD, asistentes a tres colegios de Chile.

\section{Sujetos de estudio}

Se incluyó a todos los escolares con SD, entre 6 y 18 años que asistían a dos escuelas especiales en Santiago de Chile (Capital Nacional) y a una escuela especial de la V Región (Provincia), seleccionados por conveniencia, reclutados entre junio y septiembre de 2012, cuyos padres y/o apoderados firmaron el Consentimiento Informado diseñado para este estudio. Los niños y jóvenes que pudieran hacerlo, firmaron un asentimiento informado.

\section{Definiciones}

El diagnóstico nutricional se realizó según IMC, calculado como peso en kilos y dividido por estatura en metros al cuadrado, según edad cronológica, de acuerdo a los siguientes patrones antropométricos:
1) Curva de IMC/Edad, generada sobre población con SD, realizado por Myrelid et al. (SDM/2002), en desviación estándar $(\mathrm{DE})^{11}$.

2) Curva de IMC/Edad de la NCHS $/ 2000^{12}$, según desviación estándar (DE).

3) Curva de IMC/Edad de la Organización Mundial de la Salud, OMS $/ 2007^{12}$ según percentiles.

Los niños y jóvenes fueron clasificados en desnutrición, eutrofia, sobrepeso y obesidad, según los tres patrones antropométricos, como se describe en la Tabla 1.

Se consideró "obesidad central", a aquel niño con una medición de circunferencia de cintura abdominal (CCA), mayor al percentil 90 para sexo y edad, según tablas de Fernández et $\mathrm{al}^{14}$.

Se consideró como "hábitos de alimentación inadecuada" a aquellos que refirieron consumo de menos de 4 comidas principales diarias y comer fuera de horario en las últimas $24 \mathrm{~h}$.

\section{Evaluación}

Se realizó una visita por dos médicos pediatras, a cada uno de los colegios, donde los participantes del estudio fueron pesados y medidos descalzos y con ropa interior. El pesaje, se realizó con una pesa de pie marca SECA 813 de $100 \mathrm{~g}$ de precisión y $200 \mathrm{~kg}$ de capacidad, adecuadamente calibrada; la estatura, se obtuvo con estadiómetro de $1 \mathrm{~mm}$ de precisión y 2,1 m de capacidad. Se midió CCA a todos los sujetos, con la misma cinta métrica inextensible, según la metodología utilizada en la población americana del Third National Health and Nutrition Examination Survey (NHANES III), usado como referente ${ }^{14}$. Para caracterizar a la población respecto a sus antecedentes mórbidos y sociales, se confeccionó un cuestionario de respuestas breves y recordatorio de alimentación de $24 \mathrm{~h}$, autoaplicada por los padres y/o apoderados de los niños (Anexo, Pág. 1 y 2).

\section{Registro de información y análisis estadístico}

Los datos estadísticos fueron recopilados en una planilla Excel, se calculó frecuencia de MNE y su intervalo de confianza 95\%, según cada patrón y se compararon con test de McNemar.

Las variables categóricas fueron evaluadas con $\chi^{2}$ y las continuas con test de Mann-Whitney, con $\mathrm{p}<0,05$ para significación estadística.

El análisis de concordancia entre binomios de patrones de IMC, se evaluó con el cálculo del 
Tabla 1. Clasificación del estado nutricional en niños, de acuerdo a distintos patrones de índice de masa corporal para la edad

\begin{tabular}{|clll|}
\hline Diagnóstico nutricional & SDM/2002 & NCHS/2000 & OMS/2007 \\
\hline Desnutrición & $\leq-2 D E$ & $<p 5$ & $\leq-2 D E$ \\
\hline Eutrofia & Entre -1DE y +1DE & Entre p5 y p85 & Entre -1DE y + 1DE \\
Sobrepeso & Entre +1DE y +2DE & Entre p85 y p95 & Entre + 1DE y + 2DE \\
Obesidad & $>+2 D E$ & $>$ p95 & $>+2 D E$ \\
\hline
\end{tabular}

SDM/2002: Patrón de IMC/Edad según Myrelid en población con SD ${ }^{11}$; NCHS/200: Patrón de IMC/Edad según NCHS para población general12; OMS/2007: Patrón de IMC/Edad según OMS para población general13; DE: desviación estándar; p: percentil.

coeficiente Kappa no ponderado y con ponderación lineal, según el criterio de Landis y Koch ${ }^{15-16}$, considerando las concordancias en: Pobre: 0,00; Leve: 0,01-0,20; Aceptable: 0,21-0,40; Moderada: 0,41-0,60; Considerable: 0,61-0,80; Casi Perfecta: $0,81-1,00$.

\section{Consideraciones éticas}

Este estudio respeta las normas éticas de acuerdo a la Declaración de Helsinski y fue revisado y aprobado por el Comité de ética de investigación de la Escuela de Medicina de la Pontificia Universidad Católica de Chile, el 21 de junio de 2012.

\section{Resultados}

Se obtuvo las mediciones de 81 escolares entre 6 y 18 años de edad, de los cuales 2 fueron excluidos por no tener consentimiento informado completo. De los 79 niños incluidos en el estudio $44(55,7 \%)$ eran hombres, con una mediana de edad de 12,7 años (rango: 6 a 18 años), la co-morbilidad más frecuentemente reportada fue el hipotiroidismo en $30,3 \%$ de los escolares estudiados. El 60,8\% tenía una alimentación inadecuada, según el reporte de ingesta de las últimas $24 \mathrm{~h}$. Dentro de las características de la conformación familiar, 34,7\% tenía una composición monoparental, $37,8 \%$ de las madres declaraban tener MNE y $58,1 \%$ era dueña de casa. La caracterización de la población total, diferenciada según sexo, se muestra en la Tabla 2.

La frecuencia de MNE según SDM/2002 fue de 43\% [IC 95\%: 32,7\%-54\%], según NCHS/2000 fue de 57\% [IC 95\%: 46\%-67,3\%] y para OMS/2007 fue de 65,8\% [IC 95\%: 54,9\%-75,3\%]; todas estadísticamente diferentes con un $\mathrm{p}<0,0167$. La obesidad se encontró en $21,5 \%$ de los escolares al ser evaluados con el patrón SDM/2002, en 34,2\% al usar NCHS/2000 y 37,9\% cuando se utilizó el patrón OMS/2007. La frecuencia de desnutrición, eutrofia, sobrepeso y obesidad, según los tres patrones de evaluación, se grafican en la Figura 1.

La concordancia entre los distintos patrones utilizados para el diagnóstico de MNE, de acuerdo a la valoración del coeficiente Kappa, se describen en la Tabla 3, donde se observa una considerable a casi perfecta concordancia entre OMS/2007 y $\mathrm{NCHS} / 2000$ y una menor concordancia cuando ambos, en forma independiente, se analizan con respecto a SDM/2002.

Considerando el patrón OMS/2007, 65,8\% de los escolares estudiados tenían MNE, la mediana de edad de aquellos con MNE fue de 11,7 años, siendo estadísticamente diferente a la mediana de edad de los eutróficos, según el mismo patrón de IMC. Al comparar las comorbilidades, no hubo diferencia estadística entre aquellos con MNE y los eutróficos. Todos los eutróficos según OMS/2007 también tuvieron CCA < p90, es decir, no tenían obesidad central. No hubo diferencias estadísticas entre las otras variables analizadas, sin embargo, se observa tendencia a eutrofia en aquellos con madres que refirieron haber tenido educación superior. Las características de los niños y jóvenes con MNE, comparados con aquellos considerados eutróficos, según el patrón OMS/2007, se describen en la Tabla 4 .

La frecuencia de obesidad central, medida por CCA fue de 19\%, siendo mayor en hombres versus mujeres (Tabla 2). Al evaluar la concor- 
Tabla 2. Caracterización demográfica de niños y jóvenes con síndrome de Down, según género

\begin{tabular}{|c|c|c|c|}
\hline & $\begin{array}{l}\text { Totales } \\
\text { n (\%) }\end{array}$ & $\begin{array}{c}\text { Hombres } \\
\text { n (\%) }\end{array}$ & $\begin{array}{c}\text { Mujeres } \\
\text { n (\%) }\end{array}$ \\
\hline & $79(100)$ & $44(55,7)$ & $35(44,3)$ \\
\hline Mediana de edad en meses [rango de edad en meses] & $153[72-216]$ & $144[72-216]$ & $171[77-215]$ \\
\hline Hipotiroidismo & $24(30,3)$ & $10(22,7)$ & $14(40)$ \\
\hline Cardiopatía congénita & $18(22,7)$ & $10(22,7)$ & $8(22,8)$ \\
\hline Asma & $4 \quad(5)$ & $3(6,8)$ & $1 \quad(2,8)$ \\
\hline Talla baja* & $21(26,6)$ & $8(18,2)$ & $13(37,1)$ \\
\hline Obesidad central** & $15(19)$ & $10(22,7)$ & $5(14,3)$ \\
\hline $\begin{array}{l}\text { Alimentación }(\mathrm{n}=74) \\
\text { Adecuada } \\
\text { Inadecuada }\end{array}$ & $\begin{array}{ll}29 & (39,2) \\
45 & (60,8)\end{array}$ & $\begin{array}{ll}43 & (58,1) \\
21 & (48,8) \\
22 & (51,2)\end{array}$ & $\begin{array}{rr}31 & (41,9) \\
8 & (25,8) \\
23 & (74,2)\end{array}$ \\
\hline $\begin{array}{l}\text { Composición familiar en el hogar }(n=75) \\
\text { Biparental } \\
\text { Monoparental }\end{array}$ & $\begin{array}{ll}49 & (65,3) \\
26 & (34,7)\end{array}$ & $\begin{array}{ll}42 & (56) \\
30 & (71,4) \\
12 & (28,5)\end{array}$ & $\begin{array}{ll}33 & (44) \\
19 & (57,6) \\
14 & (42,4)\end{array}$ \\
\hline $\begin{array}{l}\text { Características de las madres } \\
\text { Malnutrición por exceso }(n=74) \\
\text { Escolaridad }(n=75) \\
\text { Primaria } \\
\text { Educación superior }\end{array}$ & $\begin{array}{ll}28 & (37,8) \\
44 & (58,6) \\
31 & (41,3)\end{array}$ & $\begin{array}{ll}43 & (58,1) \\
17 & (39,5) \\
44 & (58,7) \\
25 & (56,8) \\
19 & (43,2)\end{array}$ & $\begin{array}{ll}31 & (41,9) \\
11 & (35,4) \\
31 & (41,3) \\
19 & (61,3) \\
12 & (38,7)\end{array}$ \\
\hline Ocupación de la madre $(n=74)$ & & $43(58,1)$ & $31(41,9)$ \\
\hline Dueña de casa & $43(58,1)$ & $27 \quad(62,8)$ & $16(51,6)$ \\
\hline Labora fuera de hogar & $31(41,9)$ & $16(37,2)$ & $15(48,4)$ \\
\hline
\end{tabular}

*Talla baja $=$ Talla/Edad $<-3$ DS en Patrón de Talla/Edad según OMS $2007^{13}$. **Obesidad central = Circunferencia de cintura abdominal > p90 en patrón de Circunferencia de cintura abdominal para sexo y edad, según Fernández et al ${ }^{14}$.

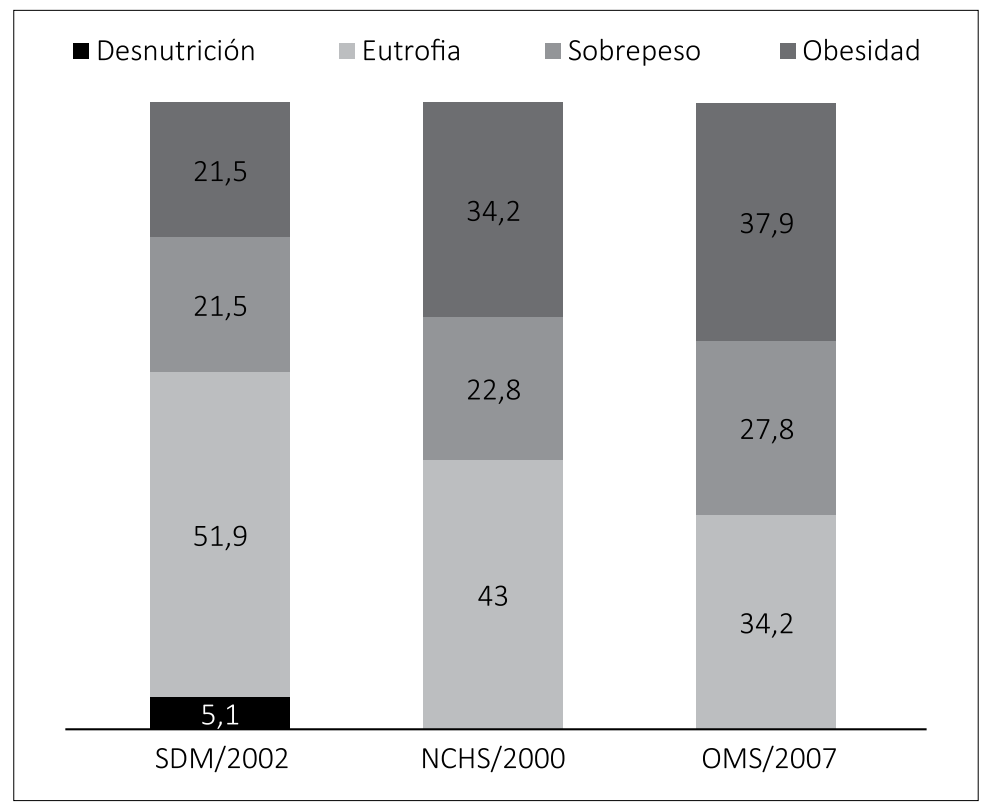

Figura 1. Prevalencia de diagnósticos nutricionales según los tres patrones de índice de masa corporal para la edad. Valores de prevalencia en \%. SDM/2002: Patrón de IMC/Edad según Myrelid en población con SD ${ }^{11}$; NCHS/2000: Patrón de IMC/Edad según NCHS para población general ${ }^{12} ;$ OMS/2007: Patrón de IMC/Edad según OMS para población general ${ }^{13}$. 
Tabla 3. Concordancia de diagnóstico de malnutrición por exceso según distintos patrones de índice de masa corporal para la edad

\begin{tabular}{|ccc|}
\hline $\begin{array}{c}\text { Comparación entre binomios } \\
\text { de patrones }\end{array}$ & Coeficiente Kappa [IC 95\%] & Kappa Lineal [IC 95\%] \\
\hline SDM/2002 vs NCHS/2000 & $0,478[0,318-0,637]$ & $0,648[0,540-0,755]$ \\
\hline SDM/2002 vs OMS/2007 & $0,349[0,189-0,510]$ & $0,552[0,440-0,665]$ \\
\hline NCHS/2000 vs OMS/2007 & $0,809[0,698-0,920]$ & $0,864[0,784-0,944]$ \\
\hline
\end{tabular}

SDM/2002: Patrón de IMC/Edad según Myrelid en población con SD ${ }^{11}$; NCHS/2000: Patrón de IMC/Edad según NCHS para población general12; OMS/2007: Patrón de IMC/Edad según OMS para población general13.

Tabla 4. Características de los niños y jóvenes con malnutrición por exceso vs eutróficos según índice de masa corporal para la edad según patrón OMS/2007

\begin{tabular}{|c|c|c|c|}
\hline & $\begin{array}{c}\text { MNE } \\
n=52(\%)\end{array}$ & $\begin{array}{c}\text { Eutrofia } \\
n=27(\%)\end{array}$ & Valor $\mathbf{p}$ \\
\hline Hombres & $30(57,7)$ & $14(51,9)$ & 0,641 \\
\hline Mediana de edad en meses [rango] & $140[72-216]$ & $174[78-215]$ & $0,048^{*}$ \\
\hline Hipotiroidismo & $16(30,8)$ & $8(32)$ & 1,000 \\
\hline Cardiopatía congénita & $14(26,9)$ & $4(14,8)$ & 0,269 \\
\hline Asma & $4 \quad(7,7)$ & 0 & 0,293 \\
\hline $\begin{array}{l}\text { CCA } \\
\qquad \begin{aligned}->p 90 \\
-<p 90\end{aligned}\end{array}$ & $\begin{array}{ll}15 & (28,9) \\
37 & (71,1)\end{array}$ & $\begin{array}{l}0 \\
27(100)\end{array}$ & 0,001 * \\
\hline $\begin{array}{l}\text { Alimentación ( } n=74) \\
\text { - Adecuada } \\
\text { - Inadecuada }\end{array}$ & $\begin{array}{ll}19 & (38,8) \\
30 & (61,2)\end{array}$ & $\begin{array}{ll}10 & (40) \\
15 & (60)\end{array}$ & 1,000 \\
\hline $\begin{array}{l}\text { Composición familiar en el hogar (n } \\
\text { - Biparental } \\
\text { - Monoparental }\end{array}$ & $\begin{array}{ll}31 & (60,8) \\
20 & (39,2)\end{array}$ & $\begin{array}{rr}18 & (75) \\
6 & (25)\end{array}$ & 0,301 \\
\hline $\begin{array}{l}\text { Característica de las madre } \\
\text { MNE }(\mathrm{n}=74)\end{array}$ & $19(38)$ & $9(37,5)$ & 1,000 \\
\hline $\begin{array}{l}\text { Escolaridad }(n=75) \\
\text { - Primaria } \\
\text { - Educación superior }\end{array}$ & $\begin{array}{ll}33 & (64,7) \\
18 & (35,3)\end{array}$ & $\begin{array}{ll}11 & (45,8) \\
13 & (54,2)\end{array}$ & 0,139 \\
\hline $\begin{array}{l}\text { Ocupación }(\mathrm{N}=74) \\
\text { - Dueña de casa } \\
\text { - Labora fuera del hogar }\end{array}$ & $\begin{array}{ll}29 & (56,9) \\
22 & (43,1)\end{array}$ & $\begin{array}{rr}14 & (60,9) \\
9 & (39,1)\end{array}$ & 0,803 \\
\hline
\end{tabular}

MNE: malnutrición por exceso; CCA: circunferencia de cintura abdominal. *Valor p significativo < 0,05.

dancia entre el diagnóstico de obesidad central, según CCA, y el diagnóstico de obesidad según los 3 patrones de IMC, se observa una concordancia considerable para los patrones SDM/2002 y NCHS/2000 y moderada para OMS/2007. Para el caso de NCHS/2000 y OMS/2007, todos los obesos centrales eran también obesos según IMC, sin embargo, la mayoría de los obesos diagnosticados según IMC, no tuvieron criterios de obesidad central (Tabla 5). 
Tabla 5. Concordancia entre obesidad diagnosticada por patrones de IMC y obesidad central según circunferencia de cintura abdominal

\begin{tabular}{|c|c|c|c|c|}
\hline \multicolumn{2}{|c|}{ Patrones de IMC } & \multirow{2}{*}{$\begin{array}{c}\text { n con obesidad } \\
\text { central* }^{*} \\
11 \\
4 \\
\end{array}$} & \multirow{2}{*}{$\begin{array}{c}\text { n sin obesidad } \\
\text { central* }^{*} \\
6 \\
58\end{array}$} & \multirow{2}{*}{$\begin{array}{c}\text { Coeficiente Kappa [IC 95\%] } \\
0,61 \quad[0,38-0,84]\end{array}$} \\
\hline SDM/2002 & $\begin{array}{l}\text { Obeso } \\
\text { No obeso }\end{array}$ & & & \\
\hline NCHS/2000 & $\begin{array}{l}\text { Obeso } \\
\text { No obeso }\end{array}$ & $\begin{array}{c}15 \\
0\end{array}$ & $\begin{array}{l}12 \\
52\end{array}$ & $0,62 \quad[0,43-0,82]$ \\
\hline OMS/2007 & $\begin{array}{l}\text { Obeso } \\
\text { No obeso }\end{array}$ & $\begin{array}{c}15 \\
0\end{array}$ & $\begin{array}{l}15 \\
49\end{array}$ & $0,55 \quad[0,35-0,76]$ \\
\hline
\end{tabular}

IMC: índice de masa corporal. *Obesidad Central = circunferencia de cintura abdominal > p90 para sexo y edad, según tablas de Fernández et al ${ }^{14}$.

\section{Discusión}

La frecuencia de MNE en la población escolar con SD estudiada es más alta de lo reportado previamente, independiente del patrón de evaluación utilizado ( $43 \%$ a $65,7 \%$ ), lo mismo si consideramos sólo obesidad, la que fue detectada en $37,9 \%$ de los escolares al usar la curva OMS/2007, superando notoriamente a lo descrito para la población general en Chile, donde la obesidad alcanza $22,1 \% 4$.

En la literatura, se postula que la causa de MNE en niños con SD incluye factores genéticos y ambientales. Dentro de los factores genéticos se describe un menor índice metabólico y niveles elevados de Leptina, lo que determinaría una resistencia a dicha hormona, menor saciedad y por lo tanto, mayor ingesta y ganancia de peso secundaria. Por otra parte, los factores ambientales, al ser modificables, son probablemente los más determinantes, como: malos hábitos alimentarios, pobre masticación, sedentarismo y una mal entendida sobreprotección que promueve conductas de refuerzos positivos a través de la comida ${ }^{17}$.

Esta alta frecuencia de MNE es preocupante, pues determina mayor riesgo bio-psico-social, especialmente descompensación de sus co-morbilidades como asma, apnea obstructiva del sueño, deterioro cardiovascular y aumento de problemas ortopédicos que asociado a la hipotonía característica del SD, perpetúan el sedentarismo, generando en los jóvenes, menor inclusión en actividades saludables de grupos coetáneos, predisponiendo a mayor discriminación y menor autoestima.

Culturalmente la obesidad de los niños y jó- venes con SD es atribuida a su condición o a sus co-morbilidades. Si bien el presente estudio no contó con evaluación bioquímica de niveles de hormonas tiroideas ni medición de repercusión cardiovascular de la obesidad, en nuestra serie, no se observó que las patologías como cardiopatía congénita, hipotiroidismo y asma, estuvieran asociadas a mayor MNE.

En niños con SD, la enfermedad tiroidea ocurre hasta en $40 \%{ }^{18}$ y si no es tratado, se relaciona con aumento de peso y obesidad a mediano y largo plazo; sin embargo, hoy en día, las recomendaciones de estudio rutinario de hormonas tiroideas y tratamiento oportuno del hipotiroidismo ${ }^{10,19}$ debieran reducir ese factor como causal de obesidad en nuestros pacientes. El asma también ha sido asociada a mayor obesidad, sin embargo, esta relación estaría determinada por la restricción de la actividad física, más que por la enfermedad misma $^{20}$.

La MNE es un factor de riesgo cardiovascular conocido; sin embargo, este riesgo es diferente para cada niño. Adicional a los antecedentes familiares, como presencia de dislipidemia, hipertensión arterial, diabetes mellitus y patologías cerebro y cardiovascular precoces ${ }^{21}$, la distribución de grasa centrípeta u obesidad central, evaluada por medio de la medición de CCA, es un conocido marcador asociado a mayor riesgo cardiovascu$\operatorname{lar}^{22,23}$. En nuestro estudio se observó que cerca de un tercio de los niños y jóvenes con MNE tuvieron criterios de obesidad central al medir su CCA, grupo que tendría mayor riesgo cardiovascular, por lo que la medición de CCA debiera ser rutinaria en la evaluación nutricional de niños con SD y así 
poder realizar intervenciones más agresivas en pro de disminuir este riesgo, más aún, teniendo en cuenta que a pesar de que la población adulta con SD tiene menor prevalencia de enfermedades ateroscleróticas cerebrovasculares, cuando ocurren, tendrían hasta cuatro veces mayor mortalidad por causa cardiovascular comparada con la población general $^{21,24}$.

Respecto a las condiciones sociales consultadas, los hábitos alimentarios de los niños y jóvenes fueron inadecuados en 60,8\% de los encuestados, $37,8 \%$ de las madres de los niños y jóvenes con $\mathrm{SD}$, declaraban tener MNE y un poco más de la mitad tenía escolaridad primaria y trabajaban en su casa; sin embargo, ninguna de esas variables fue diferente entre los niños con MNE y los eutróficos. Si bien no hubo diferencias estadísticamente significativas en el estado nutricional de los hijos de madres con estudio superiores versus primarios, sí se observó una tendencia en aquellas madres con mayor nivel educacional a tener hijos más eutróficos, lo que pudiera estar en relación con mayor conocimiento de estrategias de vida saludable, siendo el nivel educacional materno un potencial factor protector para lograr una mejor calidad de vida.

Según los patrones de IMC evaluados, las curvas para población con SD (SDM/2002) es la que pesquisa el menor porcentaje de MNE, lo que podría ser explicado en el contexto de ser una curva construida sobre una población ya obesa. Por otro lado, la evaluación según OMS/2007 detecta el mayor porcentaje de MNE. Entonces surge la pregunta, ¿el patrón SDM/2002 subestima MNE o es el patrón OMS/2007 la que lo sobreestima? Por ahora, esta pregunta no se logra responder con nuestro estudio, ya que habría que realizar evaluaciones de medición de grasa corporal y su distribución para determinarlo ${ }^{25}$; sin embargo, aunque el nivel de concordancia entre ellos es moderado a considerable, es la curva OMS/2007, la que pesquisa mayor número de niños y jóvenes con MNE y, teniendo una postura de intervención precoz y agresiva en el tratamiento de MNE, sería el patrón más adecuado para la evaluación nutricional de este grupo.

Las limitantes de este estudio son el pequeño número de niños y jóvenes incluidos, la respuesta parcial de los padres y/o apoderados de la encuesta para evaluar variables sociodemográficas y el contexto socio económico medio-bajo de las escuelas evaluadas, lo cual podría ser un sesgo al momento de evaluar esta alta pesquisa de MNE.

\section{Conclusión}

Observamos una frecuencia alarmante de MNE en niños chilenos con SD y de ellos casi un tercio con obesidad central, que conlleva a un mayor riesgo cardiovascular, por lo que los profesionales de la salud debemos educar en hábitos de vida saludable a edades tempranas, hacer evaluación nutricional adecuada, fomentar el ejercicio físico y tratamiento oportuno de co-morbilidades. Así, es importante conocer las diferencias entre los distintos patrones antropométricos usados, pues un diagnóstico precoz permitirá realizar intervenciones con un objetivo más preventivo que curativo, siempre relevante en Pediatría. Es por lo anterior que nos parece útil usar tablas de IMC según OMS/2007, en población con SD desde los 6 a 18 años, y medición de CCA en forma rutinaria, hasta que no existan tablas actualizadas y específicas para este grupo vulnerable.

Agradecimientos: Los autores agradecen a los establecimientos educacionales "Colegio Especial Armonía" de la Calera, V región-Chile, "Escuela Especial Dulce Compañía" y "Escuela Especial Padre Pío" estos últimos de Santiago de Chile, por facilitar el contacto con los niños y jóvenes que participaron de este estudio.

\section{Referencias}

1. Nazer J, Cifuentes L. Estudio Epidemiológico Global del Síndrome de Down. Rev Chil Pediatr 2011; 82 (2): 105-12.

2. Sánchez-Cruz JJ, Jiménez-Moleón JJ, Fernández-Quesada F, Sánchez MJ. Prevalence of child and youth obesity in Spain in 2012. Rev Esp Cardiol 2013; 66 (5): 371-6.

3. Vásquez F, Treviño N, Saldivar A, Vásquez C, Vásquez E. Prevalencia de Sobrepeso y Obesidad en niños de Educación Primaria: su relación con variables sociodemográficas y de salud. Revista electrónica Medicina Salud y Sociedad 2013; 3 (3): 1-24. En: www.medicinasaludysociedad.com/site/images/stories/3_3/06).pdf

4. Situación nutricional de escolares de primero básico de establecimientos subvencionados de Chile, 2011. Prevalencia de Obesidad. En: Situación nutricional escolar 
Año 2011, grupo Básica, variable Obesidad; En: bpt. junaeb.cl:8080/MapaNutricionalGx/

5. Van Gameren-Oosterom HB, Van Dommlen P, Schönbeck Y, Oudesluys-Murphy AM, Van Wouwe JP, Buitendijk SE. Prevalence of Overweight in Dutch Children with Down Syndrome. Pediatrics 2012; 130 (6): 1520-6.

6. Murray J, Ryan-Krause P. Obesity in children with Down Syndrome: Background and recommendations for management. Pediatric Nurs 2010; 36 (6): 314-9.

7. Pinheiro A, Urteaga C, Cañete G, Atalah E. Evaluación del estado nutricional en niños con síndrome de Down, según diferentes referencias antropométricas. Rev Chil Pediatr 2003; 74 (6): 585-9.

8. Retamales N, Moreno R, González A, Cerda J, Lizama M. Morbilidad y Mortalidad Durante el Primer Año de Vida en Pacientes con Síndrome de Down. Rev Chil Pediatr 2009; 80 (4): 323-31.

9. Pastor X, Quintó L, Corretger M, Gassió R, Hernández M, Serés A. Tablas de crecimiento actualizadas de los niños con Síndrome de Down. Revista internacional sobre Síndrome de Down 2004; 8: 34-46.

10. Marilyn J. Bull and the Committe on Genetics. Clinical Report- Health Supervision for Children with Down Syndrome. Pediatrics 2011; 128 (2): 393-406.

11. Myrelid A, Gustafsson J, Ollars B, Annerén G. Growth charts for Down's syndrome from birth to 18 years of age. Arch Dis Child 2002; 87 (2): 97-103.

12. Kuczmarski RJ, Ogden CL, Guo SS, Grummer-Strawn LM, Flegal KM, Mei Z, et al. 2000 CDC growth charts for the United States: Methods and development. National Center for Health Statics. Vital Health Stat11 2002; (246): 1-190.

13. De Onis M, Onyango AW, Borghi E, Siyam A, Nishida C, Siekmann J. Development of WHO growth reference for school-aged children and adolescents. Bulletin of the World Health Organization 2007; 85: 660-7.

14. Fernández JR, Redden DT, Pietrobelli A, Allison DB. Waist Circumference percentiles in nationally representative samples of African-American, European-American and Mexican-American children and adolescent. J Pediatr 2004; 145 (4): 439-44.
15. Landis JR, Koch GG. The measurement of observer agreement for categorical data. Biometrics 1977; 33 (1): 159-74.

16. Cerda J, Villarroel L. Evaluación de la concordancia inter-observador en investigación pediátrica: Coeficiente de Kappa. Rev Chil Pediatr 2008; 79 (1): 54-8.

17. Murray J, Ryan-Krause P. Obesity in children with Down Syndrome: Background and recommendations for management. Pediatric Nurs 2010; 36 (6): 314-9.

18. Gibson PA, Newton RW, Selby K, Price DA, Leyland K, Addison GM. Longitudinal Study of thyroid function in Down's syndrome in the first two decades. Arch Dis Child 2005; 90 (6): 574-8.

19. Lizama M, Retamales N, Mellado C. Recomendaciones de cuidados en salud de personas con síndrome de Down: 0 a 18 años. Rev Med Chile 2013; 141 (1): 80-9.

20. McDowell KM, Craven DI. Pulmonary complications of Down syndrome during childhood. J Pediatr 2011; 158 (2): 319-25.

21. Day SM, Strauss DJ, Shavelle RM, Reynolds RJ. Mortality and causes of death in persons with Down syndrome in California. Dev Med Child Neurol 2005; 47 (3): 171-6.

22. de Ferranti SD, Gauvreau K, Ludwig DS, Neufeld EJ, Newburger JW, Rifai N. Prevalence of the metabolic syndrome in American adolescents: Findings from the Third National Health and Nutrition Examination Survey. Circulation 2004; 110 (16): 2494-7.

23. Bancalari R, Díaz C, Martínez-Aguayo A, Aglony M, Zamorano J, Cerda V, et al. Prevalencia de hipertensión arterial y su asociación con la obesidad en edad pediátrica. Rev Med Chile 2011; 139 (7): 872-9.

24. Hill DA, Gridley G, Cnattingius S, Mellemkjaer L, Linet $\mathrm{M}$, Adami HO, et al. Mortality and cancer incidence among individuals with Down syndrome. Arch Intern Med 2003; 163 (6): 705-11.

25. Urrejola P, Hernández M, Icaza M, Velandia S, Reyes M, Hodgson M. Estimación de masa grasa en niños chilenos: ecuaciones de pliegues subcutáneos versus densitometría de doble fotón. Rev Chil Pediatr 2011; 82 (6): 502-11. 